\title{
BRAZILDAM: A BENCHMARK DATASET FOR TAILINGS DAM DETECTION
}

\author{
Edemir Ferreira $^{1}$, Matheus Brito ${ }^{1}$, Remis Balaniuk ${ }^{2}$, Mário S. Alvim ${ }^{1}$, Jefersson A. dos Santos $^{1}$ \\ ${ }^{1}$ Department of Computer Science, Universidade Federal de Minas Gerais, Belo Horizonte, MG, Brazil - CEP $31270-901$ \\ ${ }^{2}$ Universidade Católica de Brasília and Tribunal de Contas da União, Brasília, DF, Brazil - CEP 70042-900 \\ \{edemirm, msalvim, jefersson\}@dcc.ufmg.br, matheusb@eng.grad.ufmg.br, remisb@tcu.gov.br
}

KEY WORDS: Tailings Dam Detection, Remote Sensing, Deep Learning

\begin{abstract}
:
In this work we present BrazilDAM, a novel public dataset based on Sentinel-2 and Landsat- 8 satellite images covering all tailings dams cataloged by the Brazilian National Mining Agency (ANM). The dataset was built using georeferenced images from 769 dams, recorded between 2016 and 2019. The time series were processed in order to produce cloud free images. The dams contain mining waste from different ore categories and have highly varying shapes, areas and volumes, making BrazilDAM particularly interesting and challenging to be used in machine learning benchmarks. The original catalog contains, besides the dam coordinates, information about: the main ore, constructive method, risk category, and associated potential damage. To evaluate BrazilDAM's predictive potential we performed classification essays using state-of-the-art deep Convolutional Neural Network (CNNs). In the experiments, we achieved an average classification accuracy of $94.11 \%$ in tailing dam binary classification task. In addition, others four setups of experiments were made using the complementary information from the original catalog, exhaustively exploiting the capacity of the proposed dataset.
\end{abstract}

\section{INTRODUCTION}

On 25 January 2019 a tailings dam at the Córrego do Feijão iron ore mine in Brumadinho, Brazil, suffered a catastrophic slope failure, followed by a mudflow that killed at least 248 people. This tragic event, three years and two months after the rupture of another large tailings dam in Mariana, which killed 19 people and destroyed the village of Bento Rodrigues, has resurrected the ghost of disasters that precarious structures can cause.

A tailings dam is typically an embankment used to store by products of mining operations. Tailings can be liquid, solid, or a mixture of fine particles suspended in liquid, usually toxic and potentially radioactive. Solid tailings are often used as part of the structure itself. These impoundments are designed for permanent containment and are between the largest man-made structures on Earth (Morgenstern, 2001).

The number of tailings dam failures has doubled in the past 20 years. Advances in mining technology have made it possible to exploit lower grade deposits despite decreasing commodity prices, which means disposing of more rejects and putting more pressure on tailings facilities (Armstrong et al., 2019). Failure of tailings dams can be catastrophic, rapidly releasing large amounts of water and solid material, potentially causing large loss of life and huge damages to the environment and property. The risks, the challenges for long-term containment and the relatively poor safety-record revealed by the numbers of failures in tailings dams have led to an increasing awareness of the need for enhanced safety provisions (ICOLD, 2001).

There is no complete inventory of active tailings impoundments around the world. The lack of any comprehensive tailings dam database has prevented meaningful analysis of the technical failures that could help prevent future incidents. The records are very incomplete on crucial data elements: design height of dam, design footprint, construction type (upstream, downstream, center line), age, design life, construction status, ownership status, capacity, release volume, runout, etc (Yilmaz, Fall, 2017). Risk management and early-warning of dangerous trends, like accelerating displacements of slopes, are essential to support decision making, but require frequent high quality data.

Remote-sensing has been increasingly used to build monitoring applications. The identification of precursors to catastrophic slope failures on tailing dams from space was proved possible using Interferometric Synthetic Aperture Radar (InSAR) (Carlà et al., 2019). Satellite images can also be used in combination with other sources of information in order to assess risk or investigate specific circumstances on tailings dams collapses (Williams, Strydom, 1999). Remote-sensing image analysis in combination with machine learning methods have seen a massive rise in popularity for over the past few years. Machine learning has been applied to tasks including image fusion, image registration, scene classification, object detection, land use and land cover (LULC) classification, segmentation, and object-based image analysis (OBIA) (Ma et al., 2019). The use of these combined technologies can be very useful for the investigation of issues concerning the tailings dams. Scene classification and image segmentation could be used to acquire comprehensive tailings dam databases, including footprints, heights, volumes, changes throughout time and contained waste classification. These databases could be used for risk assessment and monitoring by regulatory agencies and local communities.

Machine learning methods require rich training datasets in order to fit, evaluate and test predicting models. The acquisition and preparation of these datasets can be arduous or even impracticable if there are no trustful sources of ground truth information.

In order to facilitate and stimulate the use of machine learning methods on the research on tailings dams issues, we have built a dataset, named BrazilDAM, based on Sentinel-2 and Landsat- 8 satellite images covering all tailings dams cataloged in Brazil.

In this paper, we present our dataset ${ }^{1}$, and show preliminary

${ }^{1}$ http://www.patreo.dcc.ufmg.br/brazildam-dataset/ 
results on the use of deep learning methods for the discovery of tailings dams on large area satellite images.

\subsection{Motivations}

Brazil is one of the main mineral exporters in the world. Mining accounts for almost 7\% of Brazil's GDP and generates hundreds of thousands of jobs, making it one of the most important aspects of national development and economic stability. Despite its importance, mining activity has caused severe environmental impact. Among the different environmental issues, mining activities has caused landscape degradation, erosion, soil contamination, groundwater and surface water pollution (IBRAM, 2017). According to a report by the Brazilian National Water Agency $\mathrm{ANA}^{2}$, there are 780 mining tailings dams in Brazil, which are part of the 24,092 dams cataloged by the agency.

According to the report released by ANA, referring to 2017, only $42 \%$ of the known dams are officially licensed. The lack of comprehensive information forbids the proper risk classification of $76 \%$ of all dams. The inspection of the dams is responsibility of 39 different regional and four Brazilian federal government agencies. However, most of regional agencies have no official assigned team to work on dam safety control. This is the case of state of Minas Gerais, where the most recent tragedies occurred. As a consequence, in 2017 only $3 \%$ of the all catalogued dams were visited by the supervisory bodies. Compromised structures were detected in 45 of the visited dams. ANA centralizes the data reported by the inspection bodies and is supposed to maintain and share the government official dams database. Nevertheless, the lack of vital information is evident. For instance, 18,446 dams have no height information, 9,584 have no capacity information, and 18,663 dams have not been classified for their potential damage. Without this information it is not possible to identify risky dams that should be closely monitored. There is also evidence of a probably large number of uncatalogued dams. In fact, Minas Gerais State Secretariat of Environment and Sustainable Development - SEMAD, responsible for auditing dams in that state, reported in 2017 the existence of 57 dams, a number much lower than the 698 structures registered in the State of Minas Gerais Environmental Foundation Dam Database.

The depicted scenario points to the need for better quality information and better monitoring instruments concerning the tailings dams in Brazil. The recent technologies on remote sensing and machine learning can be used to create helpful solutions to be used by the public administration and its supervisory bodies but also by local communities and non governmental organizations.

\subsection{Challenges and Contributions}

In this paper, we claim the following contributions: (1) we introduce a novel public dataset based on Sentinel-2 and Landsat8 satellite images covering all tailings dams cataloged in Brazil; and (2) we provide a benchmark addressing the challenge of landuse and land-cover classification for the proposed BrazilDAM dataset using several modern $\mathrm{CNN}$ architectures.

\section{DATASET ACQUISITION}

The Brazilian National Mining Agency makes available on its website a georeferenced database describing all officially registered tailings dams in the country (DNPM, 2019). The database contains some identification information about the dams

${ }^{2} \mathrm{https} / / /$ www.ana.gov.br/noticias/45-barragens-preocupam-orgaosfiscalizadores-aponta-relatorio-de-seguranca-de-barragens-elaboradopela-ana/rsb-2017.pdf/view ; Accessed 29-November-2019 and their owners and a spatial coordinates of a point indicating each dam's location. There is also some technical information like the main ore, height, volume, constructive method, risk category, potential damage associated with the dam.

The main providers of open access satellite imagery are NASA and the European Space Agency (ESA), with the CopernicusSentinel program and the Landsat Mission respectively. Both of the satellites used, Sentinel-2 and Landsat 8, are sun-synchronous. They capture images with Multispectral Imager (MSI), however, exist differences between the resolution and the wavelength of each band.

To deal with Landsat and Sentinel-2 image collections we chose to use the Google Earth Engine platform. The Google Earth Engine (GEE) (Gorelick et al., 2017) is a cloud computing platform designed to store and process huge datasets. The easily accessible and user-friendly front-end provides a convenient environment for interactive data and algorithm development. Google archived all the Landsat and Sentinel image collection and linked them to the cloud computing engine for open source use. Besides to provide the computational infrastructure and the image collections, the GEE API allows the images processing to deal with some commons problems, as cloud cover.

According to (ESA, 2000-2019) the 13 spectral bands of Sentinel2 range from the Visible (VNIR) and Near Infra-Red (NIR) to the Short Wave Infra-Red (SWIR). Also has three bands that measure atmospheric effects (aerosols, cirrus, and water vapor). Sentinel's resolutions for RGB are 10 meters per pixel. This satellite can cover the entire world in five days, and generate a temporal ax of multi-spectral images.

Still, according to (Masek, 2019), the 11 spectral bands of Landsat 8 are collected in a different resolution ( 30 meters per pixel in RGB) and wavelength range, although there is an intersection between then. Landsat 8 can cover the entire globe every sixteen days, doing 223 orbit cycle.

All this information available freely allows us to create a new dataset that covers all dam labeled. Was used all bands of both satellites and an annual time-series from January 1st, 2016 until September 5th, 2019 (the date that our algorithm starts running).

\subsection{Satellite Image Acquisition}

To deal with this amount of data of both satellites, we choose to use the Google Earth Engine. The engine works with a data structures based on requests, the Image Collection is a stack of images based in Earth Engine collection ID. This structure allows operations like filtering, mapping, reducing, compositing and iterating. Regarding the Image structure, are a raster composed of one or more bands that withhold their name, data type, scale, mask, and projection.

For image acquisition, we use the COPERNICUS/S2 Image Collection for Sentinel and LANDSAT/LCO8/CO1/T1_TOA for Landsat. As can be seen a workflow, in Figure 1, after choosing the satellite some filters were made to improve the overall quality.

The years were separated into four different folders, using the date filter images of the years 2016, 2017, 2018 and 2019 were obtained. This was done to create the time axis in the dataset.

Then, a boundary filter delimits the context of image acquisition. A square geometry was made, leaving the point coordinate 


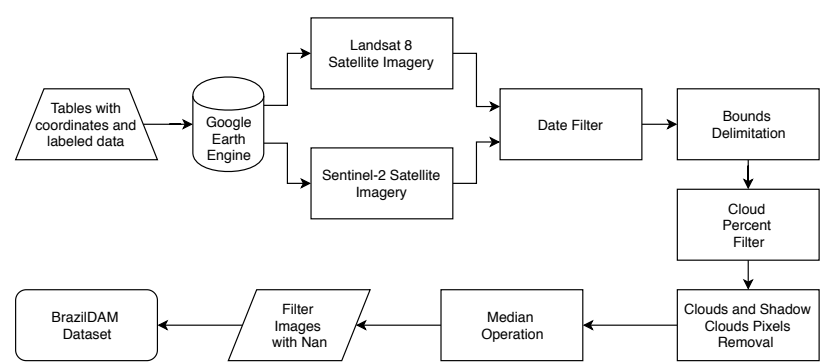

Figure 1. Workflow that illustrates the image acquisition.

exactly in the center of the image. The distance between the center and the closest borders are 1.9 kilometers, this resulted in images with $384 \times 384$ pixels.

Using the metadata of the images, it was also possible to filter the percentage of clouds within the images. This makes possible to present the features more easily.

To improve the quality, a function was mapped in each of the images of the collection, which removed all the pixels that were marked as a cloud by the atmospheric context bands (QA60 for the Sentinel and BQA in Landsat). Finally, to return a raster Image was made a median operation between the images of the Image Collection.

This operation, depicted in Equation 1, is based on the assumption that clouds are not in the same place permanently. Therefore, most parts of pixels in the same region will not have clouds. In making the median we look for the most common pixel value, which will be closest to the actual pixel value in that region. This operation is done individually in each of the bands.

$$
\rho\left(x_{j}\right) \approx \operatorname{median}\left(\rho\left(x_{1,2,3, \ldots, k}\right)\right)
$$

where

$$
\begin{aligned}
& \rho\left(x_{j}\right)=\text { the observed band of interest } \\
& x=\text { image with year delimitation } \\
& j=\text { the } \mathrm{j} \text { th pixel } \\
& k=\text { is the total number of pixels }
\end{aligned}
$$

With all the images of that year, the demarcated context area, no cloud pixels and the median operation performed are generate the final image. However, we face some problems. By filtering the amount of cloud by lest then $30 \%$, some of the collections returned no images. Some regions in Brazil are very humid and have clouds for much of the year. To deal with this limitation, we made an algorithm that increases this percentage by $10 \%$ until they're at least one image.

Another problem was some medians returning NaN (not a number). This was due to the removal of the flagged pixels with clouds, all pixels on that time axis were removed. For these cases, the median was calculated without the cloud removal function. Even so, some requests did not return any images. In this case, some folders were left with fewer files.

For the not-dam locations, where was used the coordinate above and under each clip. This can be seen in Figure 2(a), where the red square is the clip of a dam and in blue and green are the clips of not dams. To ensure that none of the not dam label contains a dam in itself, we delete the images where exists an intersection with the base location of the dam as can be noticed in Figure 2(b).

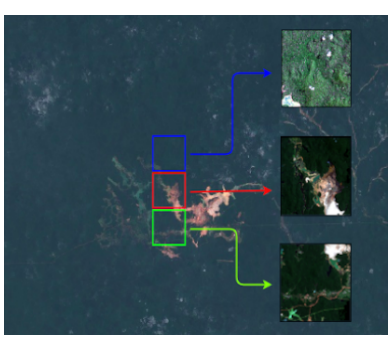

(a) Not dam acquisition.

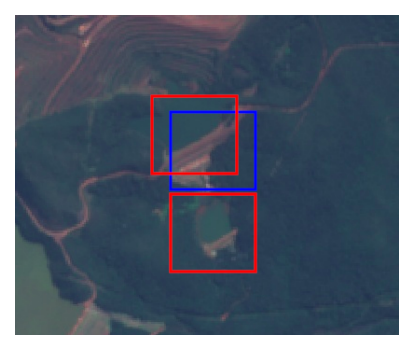

(b) Intersection issue.
Figure 2. Explanation of not dam images acquisition and intersection issues.

It is important to attend that some images get noised, or neither were added to the dataset. The reasons of those were the impression of data coordinates, few quality images in a year or artifacts created by the median operation. As are shown in Figure 3, where the 3(a) there is an example of failure in the satellite acquisition and in 3(b) we have some artifacts created by the median when a mosaic was necessary.

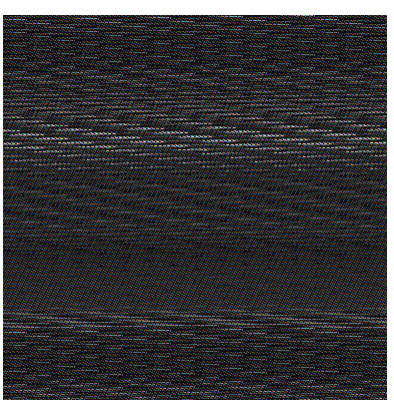

(a) High noise image.

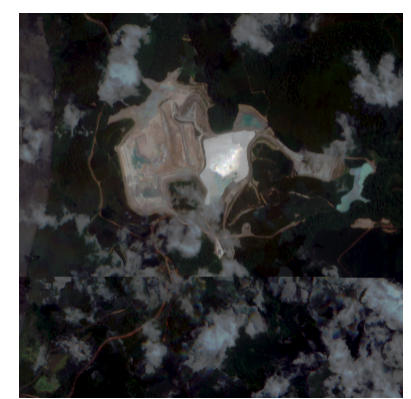

(b) Mosaic image.
Figure 3. Examples of bad images in acquisition of dams.

\section{DATASET BENCHMARKING}

To establish a benchmark for the proposed dataset, experiments was done using state-of-the-art deep learning networks in the following tasks: Dam classification, Ore classification, Constructive method, Risk category and Associated Potential Damage.

For each of the tasks performed, only images extracted from sentinel satellite in the year 2019 were used. To statistically validate the classification results, the cross-validation procedure was used, where the data used for training and testing are rotated to simulate the results in an independent data set. Without loss of generality, the results and discussions can be extended to the rest of the data. In order to fully exploit the data, six different neural network architectures were used for each classification task: AlexNet (Krizhevsky, 2014), DenseNet (Huang et al., 2017), Inception (Szegedy et al., 2016), ResNet (He et al., 2016), SqueezeNet (Iandola et al., 2016) and VGG (Simonyan et al., 2014). For the training of the models the implementation of the PyTorch library (Paszke et al., 2017) was used. In addition, the Adam algorithm was used as optimizer in each of the networks, varying the learning rate parameter between $\lambda=\left\{10^{-2}, 10^{-3}, 10^{-4}\right\}$. Reported results were acquired by averaging the balanced accuracy across the 5 cross-validation folds. Besides, 95\% confidence intervals of the mean were also reported. In ours report tables, the first column contains the methods and learning rate that achieved the best results. The second and third columns show a mean balanced accuracy and 95\% confidence interval obtained from 5-fold cross-validation. Each of 
the following sections details the experimental protocol used to evaluate the proposed tasks, as well as the results, obtained followed by a discussion of it.

\subsection{Dam Classification}

According to ANA, a large part of the ore dams have not yet been registered or cataloged in the national safety policy. This is expensive work and requires a lot of manpower to cover the entire Brazilian territory. While there is a duty for companies to report dam construction, they often do not, causing soil contamination or disruption due to poor planning.

In the dam classification task, a total of 1925 images were used (where 769 are labeled as dams and 1156 as non-dams). The results obtained can be seen in the Table 1

\begin{tabular}{ccc}
\hline Config & A.A. (\%) & CI 95\% \\
\hline VGG 1E-04 & 93.23 & $91.65-94.80$ \\
Inception 1E-04 & 93.61 & $92.02-95.20$ \\
DenseNet 1E-04 & $\mathbf{9 4 . 1 1}$ & $\mathbf{9 2 . 3 0 - 9 5 . 9 2}$ \\
SqueezeNet 1E-04 & 89.44 & $86.57-92.31$ \\
AlexNet 1E-04 & 91.77 & $89.46-94.09$ \\
ResNet 1E-03 & 90.78 & $89.43-92.13$ \\
\hline
\end{tabular}

Table 1. This table shows the balanced accuracy of methods on the Dam Classification Task.

As can be observed in the results, the model with the largest lower level of the confidence interval was highlighted. Despite the result obtained by DenseNet, all the architectures used had results considered satisfactory by the authors. In order to better understand the misclassifications of the generated model, we highlight two examples of incorrectly classified samples. Examples of false positive and negative can be seen in Figure 4.

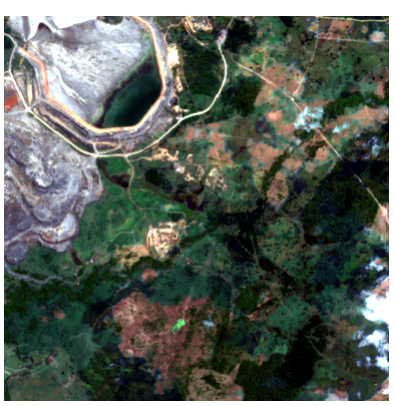

(a) False Negative

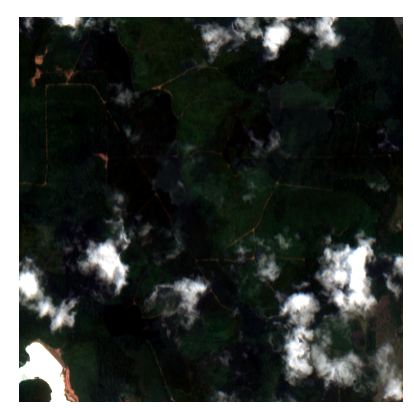

(b) False Positive
Figure 4. Examples of dam and non-dam samples incorrectly labeled. Images (a) is false negative (dam image predicted as non-dam) and image (b) is a false positive (non-dam image that have been classified as dam)

As can be seen from figure 4, image (a) show an example of false negative (image that is a dam but was classified as non-dam). In figure (a), although it is possible to observe a dam in the upper left corner of the image, its center is composed only of vegetation. This is a case of geographic coordinate error, where data provided by ANA do not correctly present the center of the structure. In the example of image (b), the image have poor visual quality, with the presence of artifacts or clouds.

\subsection{Ore classification}

Tailings dams are earth structures built to store mining waste. These residues are defined as the sterile fraction produced by ore extraction in a mechanical and/or chemical process that divides the crude mineral into concentrate and tailings. Although the waste is a material that does not have great economic value, its storage is crucial to avoid social and environmental impacts. In this task, the dam images were classified according to their main mining tailings. This monitoring is important to ensure that no dams are receiving deposits of other types of tailings illegally.

In this experiment, a total of 769 images of dams were used. Among the 769 dams, there are a total of 59 different types of ores. Thus to avoid unbalance between classes, only classes with at least 10 samples were selected, resulting in 15 classes. The rest of the images were assigned to the "others" class, totaling 16 classes. The results obtained can be seen in Table 2. Since in this task a multiclass classification is made, for a better interpretation of the results we can use the confusion matrix seen in Figure 2.

\begin{tabular}{lcc}
\hline \multicolumn{1}{c}{ Config } & A.A. (\%) & CI 95\% \\
\hline VGG 1E-04 & 60.22 & $54.14-66.30$ \\
Inception 1E-04 & 69.13 & $65.27-72.99$ \\
DenseNet 1E-04 & $\mathbf{7 2 . 4 2}$ & $\mathbf{6 8 . 5 5 - 7 6 . 3 0}$ \\
SqueezeNet 1E-04 & 63.71 & $59.64-67.79$ \\
AlexNet 1E-04 & 57.96 & $51.92-63.99$ \\
ResNet 1E-04 & 71.62 & $68.38-74.87$ \\
\hline
\end{tabular}

Table 2. This table shows the balanced accuracy of methods on the Ore Classification Task.

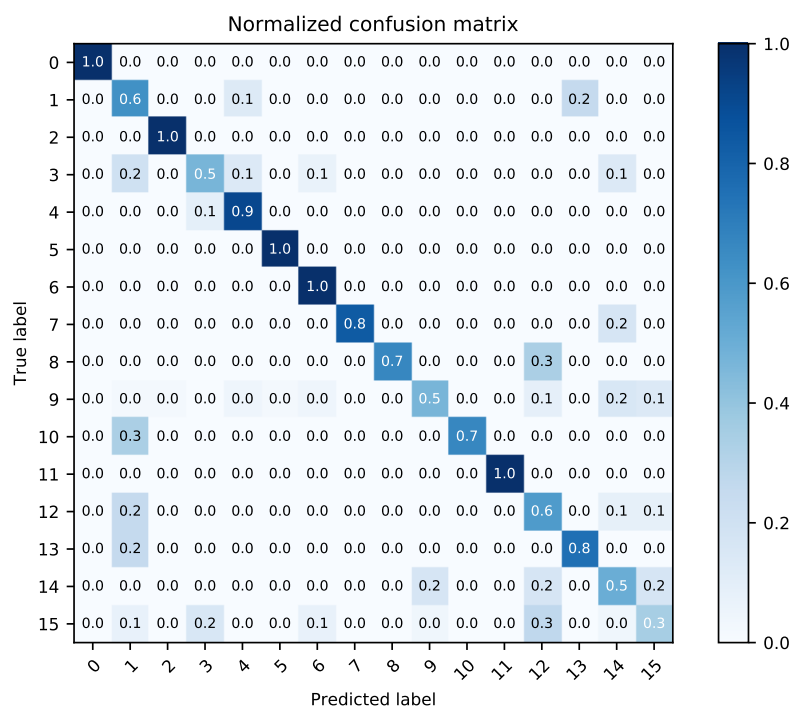

Figure 5. Confusion matrix of Ore Classification task.

Looking at Table 2, it is possible to observe again that the best result was obtained by DenseNet reaching about $72 \%$ of the balanced accuracy. However, as we can see in the confusion matrix of Figure 5, except for class 15, which represents the category "others", the model was able to successfully discriminate the different types of minerals between the dam images, considering that in a multiclass scenario, the random result is $6 \%$.

\subsection{Construction Methods}

The construction of tailings dams is directly influenced by the type of tailings to be deposited. Hydraulic embankment dams can be made from three main methods: upstream elevation, downstream elevation, and centerline method. Although all forms start from the construction of a starting dike, the difference in methods is mainly in the direction in which the elevation 
is made. The monitoring method used in its construction is extremely important to avoid the creation of dams built illegally, which are not suitable for a particular area or ore deposit.

In this task, a total of 769 images of dams were used. Among the 769 dams, there are a total of 5 different building types: Downstream method, Upstream method or unknown, Center Line method, Single-step method, Undefined. The results obtained can be seen in the Table 3

\begin{tabular}{ccc}
\hline Config & A.A. (\%) & CI 95\% \\
\hline VGG 1E-04 & 51.18 & $49.03-53.33$ \\
Inception 1E-04 & $\mathbf{5 3 . 3 0}$ & $\mathbf{5 0 . 8 1 - \mathbf { 5 5 . 8 0 }}$ \\
DenseNet 1E-04 & 55.47 & $50.41-60.54$ \\
SqueezeNet 1E-04 & 43.89 & $38.04-49.75$ \\
AlexNet 1E-04 & 49.16 & $45.97-52.35$ \\
ResNet 1E-04 & 45.25 & $44.10-46.40$ \\
\hline
\end{tabular}

Table 3. This table shows the balanced accuracy of methods on the Construction Method Task.

Looking at Table 3, it is possible to notice that all trained models achieved unsatisfactory results in the construction method classification task. Also, unlike other tasks, the results have high confidence intervals. These results can be explained by some factors, such as (1) low spatial resolution, since to distinguish the construction method,it would be necessary to obtain a spatial detailing of the dam contours; (2) the absence of height information on the sensor, the height data in the image is crucial to distinguish different declines of dam heights, and consequently its construction method; (3) dams with different construction methods in the same region, it is very common to have different tailings dams side by side but with different construction methods, as we can see in the examples in figure 6.

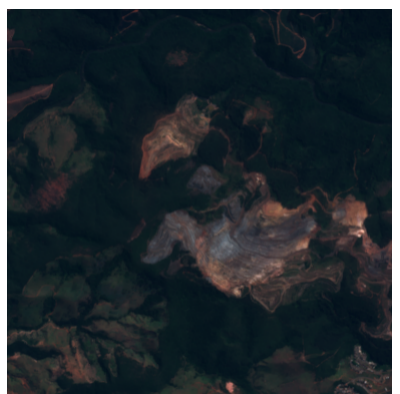

(a) Upstream method

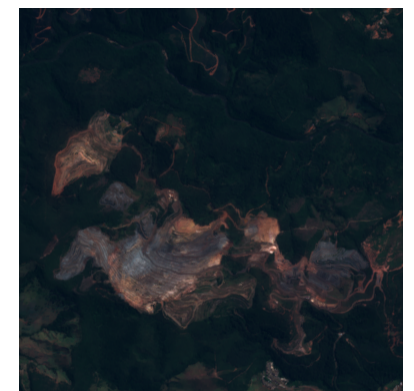

(b) Single Step method
Figure 6. Examples of dams of different construction methods within the same region. Figure (a) shows a dam constructed using the Upstream method, while in a nearby region we have the image

(b) of a dam constructed using the Single Step method.

These sets of samples end up biasing the final classification model, which receives in its training set almost identical samples with different classes, reducing its effectiveness in the test set.

\subsection{Risk Category}

The risk of dam failure is measured by considering technical and conservation characteristics. This information is provided by companies to the enforcement agency, as well as emergency action plans and periodic monitoring and conservation reports. In the case of dams that meet Política Nacional de Segurança de Barragens (PNSB) criteria, they should also receive periodic visits from enforcement agents. However, supervision in Brazil is still limited and heavily dependent on the monitoring of the

\begin{tabular}{ccc}
\hline Config & A.A. (\%) & CI 95\% \\
\hline VGG 1E-04 & 78.39 & $73.91-82.87$ \\
Inception 1E-04 & 82.91 & $79.11-86.71$ \\
DenseNet 1E-04 & $\mathbf{8 6 . 9 3}$ & $\mathbf{8 1 . 0 2} \mathbf{- 9 2 . 8 4}$ \\
SqueezeNet 1E-04 & 66.26 & $58.68-73.83$ \\
AlexNet 1E-04 & 59.41 & $55.17-63.64$ \\
ResNet 1E-03 & 83.36 & $78.99-87.72$ \\
\hline
\end{tabular}

Table 4. This table shows the balanced accuracy of methods on the Risk Category Task.

miners themselves, further increasing the risks of ore mining. (Costa, 2019)

To circumvent this manual inspection procedure, we have reformulated the problem as an image classification problem. In this case, given a subset of dam images already labeled with their risk categories, we try to predict the rest of the images using only the aerial image of the region.

In this experiment, from a total of 769 images of dams, only 423 were used. This selection was made because of the lack of label of 344 samples and 2 other images were removed to avoid the unbalance problem, since they are the only ones with the label "High". Thus given the selection, there are 423 images of dams divided between the classes: "Low", "Medium". The results obtained in this task can be seen in the Table 4.

In this experiment, it is possible to observe high average values, but with large confidence intervals as well. This is a consequence of the general criteria considered for the risk category of a dam. According to Brazilian federal dam safety legislation, risk labels take into consideration: 1) Technical characteristics, 2) Dam conservation status, 3) Safety Plan. While some technical features such as Dam Height, Dam Crown Length are easily visible in terms of images, the Dam Conservation and Safety Plan properties are not used. The absence of this information increases the uncertainty of the model.

\subsection{Associated Potential Damage}

While the risk category concerns aspects of the dam itself that may influence the likelihood of an accident, its associated potential damage is damage that may occur regardless of its likelihood of occurrence. This damage occurs due to rupture, leakage, soil infiltration or dam malfunction and can be graded according to loss of life, social, economic and environmental impacts.

In this experiment, a total of 425 images of dams were used, since the remains 344 images labels are not available. Images are divided into 3 classes of associated potential damage: Low, Medium, and High. The results obtained can be seen in Table 5

\begin{tabular}{ccc}
\hline Config & A.A. (\%) & CI 95\% \\
\hline VGG 1E-04 & 54.63 & $49.21-60.06$ \\
Inception 1E-04 & $\mathbf{6 2 . 8 7}$ & $\mathbf{5 9 . 4 3 - 6 6 . 3 0}$ \\
DenseNet 1E-03 & 57.64 & $56.25-59.03$ \\
SqueezeNet 1E-04 & 50.92 & $45.00-56.83$ \\
AlexNet 1E-04 & 55.32 & $50.77-59.86$ \\
ResNet 1E-04 & 55.96 & $51.83-60.09$ \\
\hline
\end{tabular}

Table 5. This table shows the balanced accuracy of methods on the Associated Potential Damage Classification Task.

As in the risk classification task, the complexity of this task is directly linked to the general criteria considered in the category. 
Items such as protected areas, infrastructure or services, or essential utilities equipment are hardly extracted in the form of aerial imagery. Another very important factor in this task is the consideration and existence of downstream population with a potential loss of human life. As the training of the neural network only takes into account a radius of approximately $1.9 \mathrm{~km}$ (due to the size of the image used), any housing development outside this perimeter is not considered.

\section{CONCLUSION}

In this work we introduced BrazilDAM, a novel public dataset based on Sentinel-2 and Landsat- 8 satellite images covering all tailings dams cataloged in Brazil. In addition, we provided a benchmark addressing the challenge of land-use and landcover classification using several modern $\mathrm{CNN}$ architectures in the tasks of dam classification, ore classification, constructive method, risk category and associated potential damage. Furthermore, our results suggest that deep neural networks are capable of generalizing well in some proposed tasks (Dam classification, Ore classification, Risk Category), whereas in other tasks (Construction method Associated Potential Damage) it shows poor results, possibly due to the low spatial resolution of images and contextual information considered. To the best of our knowledge, BrazilDAM is the first dataset of Brazil's tailings dams. We hope the dataset encourages the community to develop and test various data-driven algorithms to further boost the state-of-the-arts. As future work, we intend to evaluate the trained models in large scale, over the entire territorial of Brazil.

\section{ACKNOWLEDGEMENTS}

The authors would like to thank FAPEMIG, CAPES and CNPq for their financial support to this research project.

\section{REFERENCES}

Armstrong, M., Petter, R., Petter, C., 2019. Why have so many tailings dams failed in recent years? Resources Policy.

Carlà, T., Intrieri, E., Raspini, F., Bardi, F., Farina, P., Ferretti, A., Colombo, D., Novali, F., Casagli, N., 2019. Perspectives on the prediction of catastrophic slope failures from satellite InSAR. Scientific Reports.

Costa, C., 2019. Brumadinho: Brasil tem mais de 300 barragens de mineração que ainda não foram fiscalizadas e 200 com alto potencial de estrago. https://www.bbc.com/portuguese/ brasil-47056259. [Online; accessed 29-November-2019].

DNPM, 2019. Agência nacional de mineração: Cadastro nacional de barragens de mineração. http://www . anm.gov.br/assuntos/barragens/

pasta-cadastro-nacional-de-barragens-de-mineracao. [Online; accessed 29-November-2019].

ESA, 2000-2019. SENTINEL-2 Radiometric Resolutions. https://earth.esa.int/web/sentinel/user-guides/ sentinel-2-msi/resolutions/radiometric. [Online; accessed 29-November-2019].

Gorelick, N., Hancher, M., Dixon, M., Ilyushchenko, S., Thau, D., Moore, R., 2017. Google Earth Engine: Planetary-scale geospatial analysis for everyone. Remote Sensing of Environment.
He, K., Zhang, X., Ren, S., Sun, J., 2016. Deep residual learning for image recognition. Proceedings of the IEEE conference on computer vision and pattern recognition.

Huang, G., Liu, Z., Van Der Maaten, L., Weinberger, K. Q., 2017. Densely connected convolutional networks. Proceedings of the IEEE conference on computer vision and pattern recognition.

Iandola, F. N., Han, S., Moskewicz, M. W., Ashraf, K., Dally, W. J., Keutzer, K., 2016. SqueezeNet: AlexNet-level accuracy with 50x fewer parameters and $<0.5 \mathrm{MB}$ model size. arXiv preprint arXiv:1602.07360.

IBRAM, 2017. Information and Analyses on the Brazilian Mineral economy. Brazilian Mining Association - Instituto Brasileiro de Mineração (IBRAM). http://ibram.org. $\mathrm{br} / \mathrm{sites} / 1400 / 1457 / 00000380$. pdf. [Online; accessed 29November-2019].

ICOLD, U., 2001. Tailings Dams-Risk of Dangerous Occurrences, Lessons Learnt From Practical Experiences (Bulletin 121). Commission Internationale des Grands Barrages, Paris.

Krizhevsky, A., 2014. One weird trick for parallelizing convolutional neural networks. arXiv preprint arXiv:1404.5997.

Ma, L., Liu, Y., Zhang, X., Ye, Y., Yin, G., Johnson, B. A., 2019. Deep learning in remote sensing applications: A metaanalysis and review. ISPRS Journal of Photogrammetry and Remote Sensing.

Masek, J. G., 2019. Landsat Science: Landsat 8 Overview. https://landsat.gsfc.nasa.gov/landsat-8/ landsat-8-overview/. [Online; accessed 29-November2019].

Morgenstern, N. R., 2001. Geotechnics and mine waste management-update. Seminar on Safe Tailings Dam Constructions.

Paszke, A., Gross, S., Chintala, S., Chanan, G., Yang, E., DeVito, Z., Lin, Z., Desmaison, A., Antiga, L., Lerer, A., 2017. Automatic differentiation in PyTorch. NIPS Autodiff Workshop.

Simonyan, K., Zisserman, A., Zisserman, A., 2014. Very deep convolutional networks for large-scale image recognition. arXiv preprint arXiv:1409.1556.

Szegedy, C., Vanhoucke, V., Ioffe, S., Shlens, J., Wojna, Z., 2016. Rethinking the inception architecture for computer vision. Proceedings of the IEEE conference on computer vision and pattern recognition.

Williams, A., Strydom, J., 1999. A review of important and interesting technical findings regarding the tailings dam failure at Merriespruit. Journal of the South African Institution of Civil Engineering.

Yilmaz, E., Fall, M., 2017. Paste tailings management. Cham, Switzerland: Springer International Publishing. 